\title{
The Effectiveness of Aromatherapy with Lavender Essential Oil in Relieving Post Arthroscopy Pain
}

\author{
Su-Hui Huang ${ }^{1}$, Li Fang ${ }^{2}$ and Shu-Hui Fang ${ }^{3}$ \\ ${ }^{1}$ Department of Nursing, Kaohsiung Armed Forces General Hospital, Kaohsiung, Taiwan (R.O.C.) \\ ${ }^{2}$ Department of Nursing, Meiho University, Pingtung County, Taiwan (R.O.C.) \\ ${ }^{3}$ Language Education Center, Fooyin University, Kaohsiung, Taiwan (R.O.C.)
}

Correspondence should be addressed to: Li Fang; fangli72catholic36@hotmail.com

Received Date: 16 November 2013; Accepted Date: 16 March 2014; Published Date: 28 May 2014

Academic Editor: Reinaldo Nóbrega De S Almeida

Copyright @ 2014 Su-Hui Huang, Li Fang and Shu-Hui Fang. Distributed under Creative Commons CC-BY 3.0

\begin{abstract}
Research literature regarding the effectiveness of lavender essential oil aromatherapy in reducing postoperative surgical wound pain is limited and the results are inconsistent. The aim of this study was to examine the effectiveness of aromatherapy with $2 \%$ lavender essential oil via bottle necklace for postoperative arthroscopic knee wound pain relief. This was a time series experimental design study comprised of two groups. Twenty eight patients comprising the experimental group were given bottle necklaces filled with $0.5 \mathrm{ml}$ of $2 \%$ lavender essential oil, while thirty two participants of the control group were given empty bottle necklaces to wear. Aromatherapy with $2 \%$ lavender essential oil via bottle necklace effectively reduced pain for a long term (72 hours) use, and there were differences in the rate of pain decline between the experimental and control groups. There are no significant findings of groups (experimental group/control group) at 15 minutes, 4, 8, 24, and 48 hours. However, the reduction slope of pain score at the 72th hour after recovery from anesthesia in the experimental group was significantly greater than that of the control group. The pain scores of the experimental group decreased from 6.9 to 1.8, while the pain ratings of the control group decreased to 3.5 from 6.4. This approach to postoperative arthroscopic surgery knee wound pain relief is not recommended for immediate effect, but for a long term use (at least 72 hours after recovery from anesthesia). Future research should aim at establishing standards of aromatherapy care.
\end{abstract}

Keywords: Aromatherapy, lavender, arthroscopy, pain.

\section{Introduction}

Pain is the most common issue faced by post arthroscopic surgery patients, and if this pain isn't alleviated, it can delay the patient's rehabilitation program (Scott \&
Joseph, 2000). The synovial membrane, contained within the structure of the fat pad and joint capsule of the knee, is packed with free nerve endings and can create intense sensations of pain. Therefore, relieving postoperative pain is an issue that 
cannot be ignored. Current methods used to relieve postoperative knee pain include pharmaceuticals, steroids, non-steroid antiinflammatory drugs, opioids and aromatherapy (Scott \& Joseph, 2000). However, studies regarding aromatherapy's ability to alleviate postoperative arthroscopic knee pain are still limited (Barry et al., 2003).

Aromatherapy is used as an alternative treatment method in patient care, and the fat-soluble essential oils are extracted from various plant parts (Liao et al., 2008). When essential oil is used for massage, inhalation, or ingestion, it is rapidly absorbed into the bloodstream, and expelled from the body through the kidneys and liver, and carbon dioxide is exhaled (Wendy \& Jenny, 2004; Liao et al., 2008). Ergo, aromatherapy should not be implemented for treating patients afflicted by the liver or kidney dysfunction (Wendy \& Jenny, 2004; Liao et al., 2008). In recent years, healthcare workers at home and abroad have used aromatherapy for palliative patient care to improve patients' physical and psychological problems (Jeng, 2005). It is generally believed that, when the vapor of the essential oil is inhaled through the nose where it contacts with the olfactory nerve, and then the aromatic molecules are transmitted to the brain's limbic system (Wendy \& Jenny, 2004; Jeng, 2005). This triggers an emotional response which acts as an emotional regulatory force, alleviates stress, and improves hormonal coordination. Additionally, when aromatic molecules affect the hypothalamus, it affects the autonomic nervous system and the endocrine system to promote peripheral blood circulation, and regulate breathing, heart rate, and blood pressure, which in turn alleviates stress and improves hormonal coordination (Wendy \& Jenny, 2004; Jeng, 2005).

Lavender oil exhibits bactericidal, analgesic, and antispasmodic properties (Liu et al., 2008). The main components of lavender oil are linalyl acetate and linalool (Liu et al., 2008). This oil is used for treating animals as a local anesthetic. Furthermore, by inhibiting chemical pathways, linalool is used as an antispasmodic (Liu, Lin, Jiang, et al., 2008; Wendy \& Jenny, 2004).

However, some studies have shown that aromatherapy is ineffective, such as a study by Kane, et al. (2004) regarding the effectiveness of lemon and lavender oil aromatherapy in reducing patient pain during changing dressings. The research showed that aromatherapy had no effectiveness in reducing pain while the dressings were being changed, but it was effective in reducing pain after the dressing change. Perhaps it was because the fragrance of the essential oil covered the smell of the wound, and in turn, calmed and relaxed the patient.

Kim et al. (2006) carried out a study regarding the effectiveness of lavender essential oil aromatherapy in pain level reduction, pain control satisfaction and reducing the amount of analgesic used for treating breast biopsy surgery patients. The 25 patients comprising the experimental group were given oxygen masks to wear and there were two drops of $2 \%$ lavender essential oil placed inside each mask, while the members of the control group also wore oxygen masks without any lavender essential oil. At 5, 30 and 60 minutes after inhaling the essential oil, participants were asked to rate their level of pain. The results showed that inhalation of lavender essential oil did not reduce breast biopsy surgery pain, nor did it reduce the use of analgesics, however it did increase the patients' satisfaction in regards to pain control.

A study by Vakilian, Atarha, Bekhradi and Chaman (2011) was performed to explore the effectiveness of lavender oil in wound pain relief and healing. 120 primiparous women with singleton pregnancy and normal spontaneous vaginal delivery were recruited. This randomized control trial included the control group with povidoneiodine for sitz bath and the experimental group with the lavender essential oil for sitz bath, respectively. There was no significant difference between the control and experimental groups in regards to the improvement of wound pain and wound 
edema. However, the patients in the experimental group who used the lavender essential oil sitz baths showed significantly lower redness than the patients in the control group.

Dale and Cornwell's (1994) double-blind randomized study on the effectiveness of lavender essential oil relieved perineal discomfort after childbirth. There were three groups of natural-birth mothers in the study. The first group applied an extract of lavender oil as a bath additive, the second applied synthetic laboratoryproduced lavender oil, and the third and control group applied 2-methyl 3-isobutyl pyrazine. Mothers in the lavender oil group bathed in sitz baths containing 6 drops of lavender essential oil. 1.5 hours after bathing, participants were asked to rate their degree of maternal discomfort, daily for ten consecutive days. Results showed no significant difference between the three groups regarding perineum wound discomfort, and although the type of oil used had no effectiveness on pain, the use of lavender oil made the bathing experience more enjoyable.

Some studies have shown aromatherapy to be effective in pain relief or reduction in analgesic use (Alireza et. al, 2013; Rasool et. al., 2013). Alireza et. al (2013) assessed the effectiveness of inhaling Lavender essential oil on the pain relief after cesarean. The results indicated that pain degree had a significant decrease compared with the control group. Rasool et. al. (2013) investigated the effects of aromatherapy with lavender essential oil on post-tonsillectomy pain in reduction of analgesic use. The results showed that analgesic use had significant reduction.

Essential oil aromatherapy via bottle necklace is a simple and convenient way to inhale essential oil. The related aforementioned research has examined the effectiveness of essential oil inhalation and essential oil massage, but the available research literature specifically regarding postoperative arthroscopic knee wound pain is limited. Moreover, the results of the research on the effectiveness of lavender essential oil for pain reduction are inconsistent and lack consensus. Therefore, the aim of this study was to examine the effectiveness of aromatherapy with 2\% lavender essential oil via bottle necklace for postoperative arthroscopic knee wound pain relief.

\section{Methods}

\section{Operational Definition}

Essential Oils: The essential oil of this experimental group is pure $100 \%$ plant oil made by Latifa International College of Aromatherapy. The main ingredient was pure lavender essential oil at a $2 \%$ concentration.

Wound Pain: refers to the patient's subjective pain ratings by means of a visual analog scale (VAS $10 \mathrm{~cm}$ ) and pain observation table, where $10 \mathrm{~cm}$ represents severe pain, and $0 \mathrm{~cm}$ represents no pain. Patients were asked to rate pain levels according to their degrees of pain via the pain observation table in centimeters.

Anesthesia Effect Recession: the time when anesthesia had fully worn off. It means that, the time after surgery when the patient was able to lift or move his or her leg and foot under his or her own power.

\section{The Hypothesis of the Study}

Patients using lavender essential oil aromatherapy can relieve the degree of postoperative arthroscopic knee wound pain levels than those who does not use.

\section{Participants}

The following formula (World Health Organization, 1998) was used to estimate sample size: $n=2 \rho^{2}(Z 1-\alpha+Z 1-\beta)^{2} /(\mu 1-$ $\mu 2)^{2}$

(n: Sample size; Level of significance $(\alpha)$ : 5\%; Power (1- $\beta$ ): $85 \%$; $\quad$ Standard deviation $(\rho)$ of the control group: 1.2 ;

Variance $\left(\rho^{2}\right): 1.44 ; \mu 1$ : the mean of pain scores in the control group was $4.0 ; \mu 2$ : the means of pain scores in the experimental 
group were 3.0). The mean scores of pain for control group and experimental group were received from pilot study between 1 July and 31 July, 2009. Therefore, 26 for each group was the expected sample size (n) for this current study.

Sixty patients at a teaching hospital in southern Taiwan who met the inclusion criteria were selected and randomized assigned into two groups, control and experimental, for the purpose of comparison. A white ball and a black ball were put into a box. When the patients admitted to the ward, the researcher picked up a ball from the box. When the researcher assistant picked up a white ball, the patient was assigned to the experimental group. If the researcher assistant picked up a black ball, the patient was assigned to the control group. The subjects and statistician were blinded. Patients' requirements included: the ability to communicate with researchers, being willing to participate, having recently undergone arthroscopic knee surgery, not allergic to lavender, and not suffering from any form of liver or kidney dysfunction and having normal olfactory function. Patients afflicted by liver or kidney dysfunction were excluded. There are no participants with any adverse events.

\section{Designs}

These two group progressive time series experimental design and multiple treatment replication studies were carried out from May 1'st, 2010 to Jun 21st, 2010 at a regional university hospital in southern Taiwan. The intervention variable for this study was "whether or not the patient used aromatherapy with $2 \%$ lavender essential oil". Patient factors were compared for the two groups (control variables), these included gender, age, length of operation, anesthesia method, and whether or not analgesic was injected.

The researcher explained the procedures and requirements to participants, and informed consents were signed by the participants. In addition to the analgesic injections (ketofen-H 50mg q4h p.r.n) that were administered according to the patients' needs, the patients comprising the experimental group were treated with aromatherapy. The hospitalization days for patients undergoing arthroscopy were around 3 to 5 days. The effects of anesthesia receded within 72 hours of the surgery, and the examination of the effectiveness of aromatherapy with lavender essential oil inhalation on wound pain began. Due to this, the aromatherapy treatment began after the anesthesia had completely worn off. A research assistant was responsible for filling the patients' bottle necklaces with $0.5 \mathrm{ml}$ of $2 \%$ concentration lavender essential oil on a daily basis. The bottle necklaces were worn while participants were sleeping. It was sealed by bottle corks and the distance from nose to bottle was around $19 \mathrm{~cm}$ for every participants. The experimental group participants wore the bottle necklaces continuously for three days. The control group participants also wore an empty bottle necklace which is the same as the experimental group. The bottle necklace is not transparent, so that participants did not know the contents of the bottle.

\section{Instrument}

A researcher-developed data collection instrument captured data on demographics and pain assessment.

\section{Basic Personal Information}

This section includes: name, gender, age, length of operation, anesthesia method, and whether or not, analgesic was injected. This data table was created by the researchers, and filled out by the researchers while talking to the patients.

\section{Pain Assessment}

A visual analog pain scale was used for pain assessment (Visual Analogue Scale, VAS). $10 \mathrm{~cm}$, being the maximum, represents extreme pain, while zero, the scale's minimum represents no pain at all. The validity of the visual analogue scale has been confirmed by the Chinese, and has been found very reliable (Lin, Lin \& Yao, 2001). 


\section{Data Collection Procedure}

Before the examination was carried out, researchers made sure that each patient met the inclusion criteria, and willingly gave the consent. The researcher explained the procedure to the participants before the surgical procedure. After each patient was introduced to the visual analog scale (VAS), and taught how to report their degree of postoperative pain using the VAS by a research assistant. A research assistant was also responsible for monitoring when the anesthesia had fully worn off. Immediately after the anesthesia effect had receded, a research assistant gave the experimental group patients lavender essential oil bottle necklaces, and gave empty bottle necklaces to patients in the control group. The patients wore the bottles every day, for three consecutive days. A different research assistant was tasked with recording their pain ratings at the following intervals: at the time when their anesthesia had completely worn off (before patients were given bottle necklaces), then 15 minutes, 4 hours, 8 hours, 24 hours, 48 hours and 72 hours (after the patients were given bottle necklaces). The research assistant responsible for recording pain ratings was not told which participants were in which group. The participants of the control group were also evaluated at the same time as the experimental group.

\section{Ethical Considerations}

Approval of Institutional Review Board of the hospital involved was obtained. The researcher explained the procedure and requirements to participants, and informed consent were signed by the participants before undergoing surgery.

\section{Data Analysis}

The collected data was archived using Excel, and SPSS 13.0 software's ANOVA mean comparison was used for statistical analysis of variance by comparing the continuous dependant variables (age by years \& duration of surgery by minutes) of the control and experimental groups, while the Chi-Square Test was used to compare the categorical dependant variables (gender, anesthesia method, surgery complexity \& whether or not an analgesic was injected). Finally, the data was analyzed again by means of multiple linear regressions.

\section{Results}

Thirty two patients were in the control group, and twenty eight patients were in the experimental group from May $1^{\text {st }}, 2010$ to Jun 21st, 2010. Below is a comparison of the control variables of the two groups, followed by a comparison of performance metrics.

\section{Control Variables Comparison}

Control variables (gender, age, length of operation, anesthesia method, surgery complexity, and whether or not analgesic was injected) between the two groups showed no statistically significant distribution variance (Tables 1, 2), therefore, when comparing pain ratings, these variables should be controlled. The average age of participants in both groups was 35.10; average surgery duration was 83.99; men far outnumbered women (accounted for 62.5-78.57\%); and 43.75$57.4 \%$ patients did not receive an analgesic injection.

Table 1. Control Variables between the Two Groups Compared to a Continuous Variable

\begin{tabular}{|c|c|c|c|c|c|}
\hline $\begin{array}{l}\text { Variable } \\
\text { Age }\end{array}$ & Number of cases & Mean & Standard deviation & $\begin{array}{c}\text { t value } \\
1.697\end{array}$ & $\begin{array}{c}P \text { value } \\
0.095\end{array}$ \\
\hline $\begin{array}{l}\text { Control group } \\
\text { Experimental group }\end{array}$ & $\begin{array}{l}32 \\
28\end{array}$ & $\begin{array}{l}39.156 \\
31.036\end{array}$ & $\begin{array}{c}20.83 \\
15.366\end{array}$ & & \\
\hline $\begin{array}{l}\text { Surgery duration } \\
\text { Control group }\end{array}$ & 32 & 78.906 & 67.581 & -0.518 & 0.607 \\
\hline Experimental group & 28 & 89.071 & 84.429 & & \\
\hline
\end{tabular}


Table 2. Control Variable between the Two Groups Compared Variable by Category

\begin{tabular}{|l|l|c|c|c|c|}
\hline Variable & & Control & Experimental & $\chi \mathbf{2}$ value & P value \\
\hline Gender & & & & & $0.259(\mathrm{f})$ \\
\hline Female & Number & 20 & 22 & & \\
\hline Male & Rate & $62.50 \%$ & $78.57 \%$ & & \\
\hline & Number & 12 & 6 & & \\
\hline Anesthesia method & & $37.50 \%$ & $21.43 \%$ & & \\
\hline Local & Rate & & & & $0.312(\mathrm{f})$ \\
\hline & Number & 7 & 3 & & \\
\hline General & Rate & $21.87 \%$ & $10.71 \%$ & & \\
\hline & Number & 25 & 25 & & \\
\hline Analgesic injection & Rate & $78.13 \%$ & $89.29 \%$ & & \\
\hline Had injection & Number & & & & $0.437(\mathrm{f})$ \\
\hline & Rate & 18 & 12 & & \\
\hline No injection & Number & $56.25 \%$ & $42.86 \%$ & & \\
\hline & Rate & $43.75 \%$ & $57.14 \%$ & & \\
\hline
\end{tabular}

Note: f: fisher exact

\section{Comparison \\ of \\ Intervention \\ Effectiveness}

As presented in Table 3, aromatherapy with $2 \%$ lavender essential oil via bottle necklace was verified to be effective in lowering pain scores. The lowering level of pain scores in the experimental group was greater than that in the control group. Differences in pain scores at pretest did not show a statistical significance $(\mathrm{t}=0.984 ; \mathrm{p}$ $=0.326$ ) between the experimental group and the control group, which indicated that the pain scores in the two groups were not showing significant differences at first. As presented in Fig. 1 and Table 3, after the application of aromatherapy, there was not effective at the 15 th minute, 4th, 8 th, 24th, 48th, and 72nd hour after the participants of the control group recovered from anesthesia. Therefore, compared with the pain scores of the pretest (mean of pain scores $=6.4$ ), pain scores in the control group at the 15th minute, 4th, 8th, 24th, 48 th, and 72nd hour (mean of pain scores = $6.2,4.9,4.5,3.9,3.7$ and 3.5) respectively, after recovery from anaesthesia presented no significant differences $(\mathrm{t}=-0.297$,$0.620,-1.017,-0.967,-1.950$, and -3.016 ; $\mathrm{p}=0.767, \quad 0.536,0.310,0.334,0.052, \quad$ and 0.000). No significant interactions of groups (experimental group/control group) at the 15 th minute, 4 th, 8 th, 24 th, and 48th were found $(-0.297,-0.620,-1.017$, $-0.967,-1.950$ and $-3.106, p=0.767,0.536$, $0.310,0.334,0.052$ and 0.002 ). In other words, the decreasing slope of pain score in the experimental group was significantly greater than that of the control group. However, significant interactions of groups (experimental group/control group) at the 72nd hour after recovery from anesthesia was found, indicating that the decreasing slope of pain scores in the experimental group at the 72nd hour after participants recovering from anaesthesia was significantly greater than that of the control group. The pain ratings of the experimental group that was treated with aromatherapy decreased from 6.9 to 1.8 , while the pain ratings of the control group decreased to 3.5 from 6.4 . 


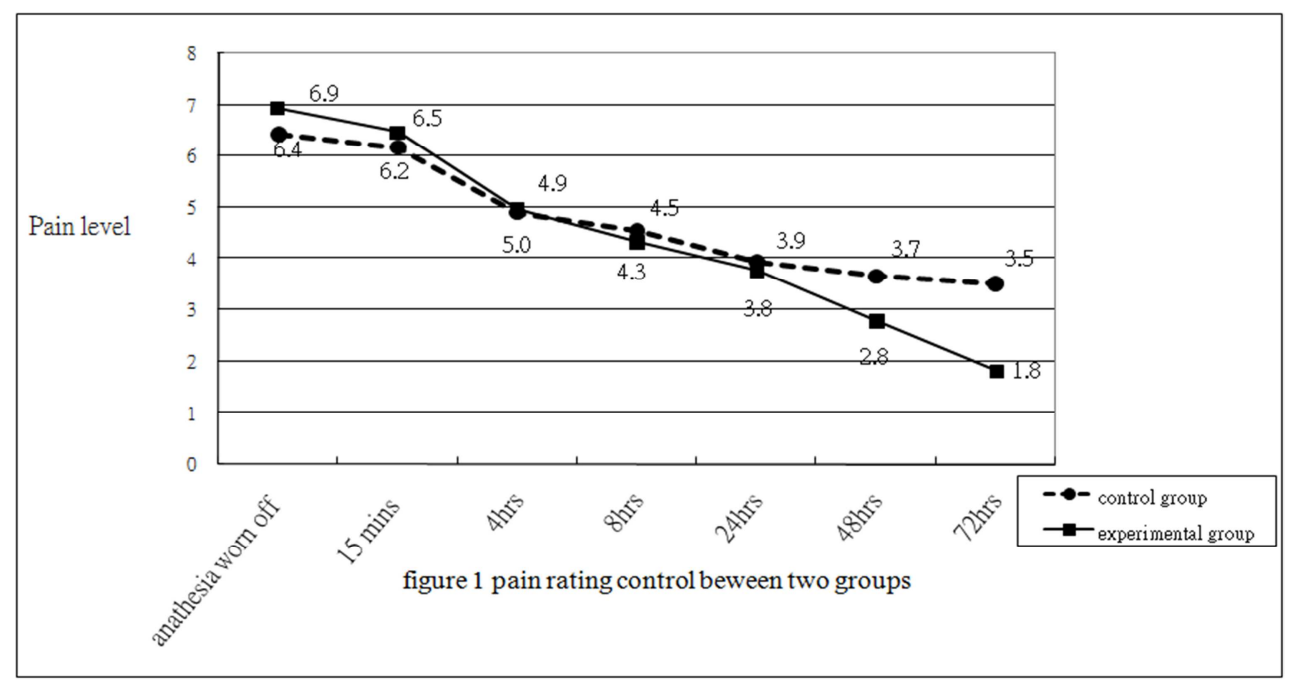

Table 3. Group Comparison between the Effectiveness of Variables

\begin{tabular}{|l|c|c|c|c|}
\hline Variable & Estimate & Std. Error & t Value & P Value \\
\hline Pre-test control group & 6.438 & 0.341 & 18.878 & 0 \\
\hline Pre-test (experimental group / control group) & 0.491 & 0.499 & 0.984 & 0.326 \\
\hline Control group (15 min. post / pre-test) & -0.256 & 0.479 & -0.534 & 0.593 \\
\hline Control group (4 hrs post / pre-test) & -1.528 & 0.479 & -3.194 & 0.002 \\
\hline Control group (8 hrs post / pre-test) & -1.892 & 0.479 & -3.953 & 0 \\
\hline Control group (24 hrs post / pre-test) & -2.498 & 0.479 & -5.22 & 0 \\
\hline Control group (48 hrs post / pre-test) & -2.771 & 0.479 & -5.79 & 0 \\
\hline Control group (72hrs post / pre-test) & -2.922 & 0.479 & -6.106 & 0 \\
\hline $\begin{array}{l}\text { Pre-test (experimental group / control group) and } \\
\text { control group (15 min. post / pre-test) interaction }\end{array}$ & -0.209 & 0.703 & -0.297 & 0.767 \\
\hline $\begin{array}{l}\text { Pre-test (experimental group / control group) and } \\
\text { control group (4 hrs. post / pre-test) interaction }\end{array}$ & -0.436 & 0.703 & -0.62 & 0.536 \\
\hline $\begin{array}{l}\text { Pre-test (experimental group / control group) and } \\
\text { control group (8 hrs. post / pre-test) interaction }\end{array}$ & -0.715 & 0.703 & -1.017 & 0.31 \\
\hline $\begin{array}{l}\text { Pre-test (experimental group / control group) and } \\
\text { control group (24 hrs. post / pre-test) interaction }\end{array}$ & -0.68 & 0.703 & -0.967 & 0.334 \\
\hline $\begin{array}{l}\text { Pre-test (experimental group / control group) and } \\
\text { control group (48 hrs. post / pre-test) interaction }\end{array}$ & -1.372 & 0.703 & -1.95 & 0.052 \\
\hline $\begin{array}{l}\text { Pre-test (experimental group / control group) and } \\
\text { control group (72 hrs. post / pre-test) interaction }\end{array}$ & -2.185 & 0.703 & -3.106 & 0.002 \\
\hline
\end{tabular}

Note: Equation: y=6.438+0.491 (groups)- 0.256 (15 min. post / pre-test)-1.528 (4 hrs post/pre-test)-1.892 ( 8 hrs post /pre-test)-2.498 ( $24 \mathrm{hrs}$ post / pre-test)-2.771 (48 hrs post / pre-test) -2.922 ( $72 \mathrm{hrs} \mathrm{post} \mathrm{/pre-test)-}$ 0.209 [both groups and control group ( $15 \mathrm{~min}$. post / pre-test) interaction ] -0.436 [ both groups and control group ( $4 \mathrm{hrs}$ post / pre-test) interaction ] -0.715 [ both groups and control group ( $8 \mathrm{hrs}$ post / pretest)interaction ] -0.680 ( both groups and control group ( $24 \mathrm{hrs}$ post / pre-test) interaction ] -1.372 ( both groups and control group ( $48 \mathrm{hrs}$ post / pre-test) interaction ] -2.185 ( both groups and control group ( $72 \mathrm{hrs}$ post / pre-test) interaction ] 


\section{Discussions}

Aromatherapy with 2\% lavender essential oil via bottle necklace was able to effectively improve postoperative arthroscopic knee wound pain levels for 72 hour use. However, there was no significant effectiveness on the short term use, which meant that duration was shorter than 72 hours. This result was consistent with the study done by Kane (2004); lemon and lavender oil aromatherapy had no immediate effectiveness on reducing pain during changing dressings, but rather subsequent to the dressing change.

Due to the fragrance of lavender essential oil, this study can hardly be considered double-blind, and its results may have been affected by the placebo effect. This will be the limitation of this study. Kim et al. (2006) carried out a study regarding the effectiveness of lavender essential oil aromatherapy in pain level reduction, pain control satisfaction and reducing the amount of analgesic used for treating breast biopsy surgery patients. Their results showed that inhalation of lavender essential oil did not reduce breast biopsy surgery pain, nor did it reduce the use of analgesics, however it did increase the patients' satisfaction in regards to pain control. Yet, an increase in pain control satisfaction is very subjective, and since most case studies are implemented by means of self-administered questionnaires that inquire about patients' subjective feelings.

\section{Conclusions}

This study proves that aromatherapy with $2 \%$ lavender essential oil via bottle necklace effectively reduced pain for the long term (72 hours) use. Therefore, due to the results of this study, this approach to postoperative arthroscopic surgery knee wound pain relief is not recommended for shorter than 72 hour use. Appropriate use of aromatherapy may improve patients' physical problems. Therefore, hospital staff managers are encouraged to include aromatherapy concepts and techniques in the continued education of nursing staff, and should aim at establishing standards of aromatherapy care.

\section{References}

Alireza, O., Kaveh, B., Reza, A., Farhad, S. \& Forough, S. (2013). "The Effect of Inhalation of Aromatherapy Blend Containing Lavender Essential Oil on Cesarean Postoperative," Anesthesia Pain, 3(1), Pp. 203-207.

Barry, S., Wallce, L. \& Lamb, S. (2003). "Cryotherapy after Total Knee Replacement: A Survey of Current Practice," Physiotherapy Research International, 8, Pp.111-120.

Dale, A. \& Cornwell, S. (1994). "The Role of Lavender Oil in Relieving Perineal Discomfort Following Child Birth: A Blind Randomized Clinical Trial," Journal of Advanced Nursing, 19, Pp.89-96.

Jeng, Y. X. (2005). 'The Application of Aromatherapy in Nursing,' Nursing Magazine, 52, Pp.11-15.

Kane, F. M. A., Brodie, E. E., Coull, A., Coyne, L., Howd, A., Milne, A., et al. (2004). "The Analgesic Effect of Odour and Music upon Dressing Change," British Journal of Nursing, 13, Pp.S4-S12.

Kim, J. T., Wajda, M., Cuff, G., Serota, D., Schlame, M., Axelrod, D. M. \& Guth, A. A. (2006). "Evaluation of Aromatherapy in Treating Postoperative Pain: Pilot Study," Pain Practice, 6, Pp.273-277.

Laio, L. C.,Kuo, H. W., Wang, M. T.,Haung, H. H. \& Liu, P. E. (2008). 'The Knowledge, Attitude, Behavior and Related Factors toward Aromatherapy among Nurses,' MidTaiwan Journal of Medicine, 13(4), Pp.200207.

Lin, P. C., Lin, C. C. \& Yao, E. (2000). 'Pain Control after Spinal Surgery - (Every Four Hours When Necessary the Effect of Intravenous Infusion of Demerol,' Nursing Magazine, 48, Pp.49-57. 
Liu, W. E., Lin, L. J., Jiang, Y. Y., Jhen, J. Y., Lin, P. X., Jien, Y. X. \& Lin, W. F. \& Lin, R. X. (2008). 'Essential Oil Massage Effects on Neck and Shoulder Pain,' Nursing Leadership, 9, Pp.18-30.

Rasool, S., Saeed, S., Valiollah, H., Gholamreza, A., Mahdi, B. \& Mahdi, M. (2013). "Evaluation of the Effect of Aromatherapy with Lavender Essential Oil on Post-Tonsillectomy Pain in Pediatric Patients: A Randomized Controlled Trial," International Journal of Pediatric Otorhinolaryngology, 77, Pp. 1579-1581.

Scott, S. R. \& Joseph, S. (2000). "Current Concepts Review: Pain Management in Patients Who Undergo Outpatient Arthroscopic Surgery of the Knee," Journal of Bone and Joint Surgery, 82, Pp.17541766.
Vakilian, K., Atarha, M., Bekhradi, R. \& Chaman, R. (2011). "Healing Advantages of Lavender Essential Oil during Episiotomy Recovery: A Clinical Trial," Complementary Therapies in Clinical Practice, 17, Pp.50-53. Publisher - Google Scholar

Wendy, M. J. \& Jenny, M. W. (2004). "Aromatherapy Practice in Nursing: Literature Review," Journal of Advanced Nursing, 48, Pp.93- 103.

World Health Organization (1998). 'Sample Size Determination in Health Studies,' Version 2.0, Singapore: KC Lune \& Peter Chiam. 